ఠ

\title{
Axitinib for the treatment of patients with advanced metastatic renal cell carcinoma (mRCC) after failure of prior systemic treatment
}

This article was published in the following Dove Press journal:

OncoTargets and Therapy

16 June 2012

Number of times this article has been viewed

Viktor Grünwald'

Axel S Merseburger ${ }^{2}$

'Clinic for Hematology, Hemostasis, Oncology and Stem Cell

Transplantation, Hannover Medical School, ${ }^{2}$ Department of Urology,

Hannover Medical School,

Hannover, Germany
Correspondence: Viktor Grünwald

Clinic for Hematology, Hemostasis,

Oncology and Stem Cell Transplantation,

Hannover Medical School, Carl-Neuberg-

Str I, 30625 Hannover, Germany

Tel +495II 5324077

Fax +495II 5328077

Email gruenwald.viktor@mh-hannover.de
Abstract: The landscape of renal cell carcinoma (RCC) treatment has changed dramatically during recent years. Bevacizumab/interferon, sunitinib, sorafenib, temsirolimus, everolimus, and pazopanib have been proven effective in metastatic RCC. Axitinib is a novel tyrosine kinase inhibitor, which inhibits the vascular endothelial growth factor receptor (VEGFR) at subnanomolar level. Based on this extraordinary VEGFR inhibition, axitinib is considered a next-generation agent. The recent AXIS trial reported on axitinib's efficacy in second line treatment of RCC, which led to its recent approval in the USA. This review focuses on the clinical efficacy of axitinib in RCC patients.

Keywords: tyrosine kinase inhibitor, axitinib, tivozanib, renal cell carcinoma, VEGF

\section{Introduction}

The landscape of therapeutic options in metastatic renal cell carcinoma (mRCC) has changed dramatically during recent years. The introduction of targeted therapies has had a major impact on therapeutic efficacy. Inhibitors of the vascular endothelial growth factor (VEGF) receptor, ${ }^{1-3}$ or mammalian target of rapamycin (mTOR), ${ }^{4,5}$ represent the backbone of current palliative therapies. ${ }^{6}$ With the availability of therapeutic diversity, sequential therapies have already been implemented in the clinical treatment algorithm. The mTOR inhibitor (mTORi) everolimus was the first agent that showed Phase III RECORD-1 data, with a superior progression-free survival (PFS) outcome, compared to placebo, ${ }^{5}$ and is considered a valuable treatment option in VEGF-resistant disease. Numerous patient series and early clinical trials suggested treatment efficacy of the subsequent use of inhibitors of the VEGF receptor (VEGFR) ${ }^{7-9}$ Axitinib is a novel VEGFR inhibitor, which achieved superior progression-free survival in second-line therapy, compared to sorafenib, which is another tyrosine kinase inhibitor (TKI), in mRCC. ${ }^{10}$ These studies leave the clinical landscape with the remaining question of what is the best therapeutic choice in resistant disease. An ongoing study explores sorafenib in comparison with the mTOR inhibitor temsirolimus, and may help to elucidate the role of TKI versus mTORi in second-line therapy, with respect to sequential usage.

This review explores the role of the novel VEGFR inhibitor axitinib in the current landscape of mRCC.

\section{Current treatment of metastatic RCC}

The landscape of mRCC treatment changed irreversibly with the approval of sunitinib and sorafenib in 2006. These TKIs pioneered the concept of VEGF-targeted therapies 
in $\mathrm{mRCC}$, which was based on insights into the molecular mechanisms of carcinogenesis in RCC. Loss of function of the von Hippel-Lindau (VHL) gene has been identified as a key step in tumor development in RCC, with clear cell histology. ${ }^{11,12}$ Restoration of VHL function was shown to be associated with tumor response in xenograft models. ${ }^{13} \mathrm{Clear}$ cell RCC is predominantly found among renal carcinomas, and is extensively explored in clinical trials.

Current recommendations for $\mathrm{mRCC}$ treatment employ VEGF-targeted agents and mTORis, mainly driven by Phase III data. ${ }^{14,15}$ Depending on individual risk category, a choice of first line options may be given (Table 1). Upon disease progression, another line of therapy may be given based on Phase III and Phase II data, including TKIs, after cytokine failure, and everolimus, after failure of VEGF inhibitors (Table 1).

Exposure to VEGF-targeted agents is associated with an improvement in PFS of 10.2-11.1 months, in treatment-naïve patients, and has been reported to achieve an overall survival (OS) of 22.9-26.4 months (Table 2). The aforementioned trials included a high proportion of subsequent therapies, which may have had an impact on OS in the current series. In poor-risk patients, the clinical outcome is far worse, and temsirolimus remains the only agent, with Phase III data, designed for this cohort.

Multiple studies explored the role of subsequent therapies in patients with refractory disease (Table 3 ). Prolonged inhibition of the VEGF axis has been shown to be effective in patients with failure to bevacizumab, sorafenib, or sunitinib. ${ }^{7,9,16}$ Changes in the mode of action, and introduction of an mTORi, in resistant disease were also reported to achieve clinical benefit. ${ }^{17-19}$ Based on such findings, the Phase III RECORD-1 study was launched, and determined the efficacy of everolimus in patients with $\mathrm{mRCC}$, resistant to VEGF-targeting agents. ${ }^{5}$ Only $2 \%$ achieved a partial

Table I Treatment algorithm for palliative therapy in metastatic renal cell carcinoma ${ }^{14,15}$

\begin{tabular}{llll}
\hline Setting & Risk category & Therapy $^{\mathrm{a}}$ & Options $^{\mathrm{b}}$ \\
\hline First line & $\begin{array}{l}\text { Favorable or } \\
\text { intermediate risk }\end{array}$ & $\begin{array}{l}\text { Sunitinib } \\
\text { Bevacizumab }+ \\
\text { interferon- } \alpha\end{array}$ & $\begin{array}{l}\text { High-dose }_{\text {interleukin-2 }} \\
\end{array}$ \\
& Poor risk & $\begin{array}{l}\text { Pazopanib } \\
\text { Temsirolimus }\end{array}$ & Sunitinib \\
Second line & Prior cytokine & Sorafenib & Sunitinib \\
& Prior VEGF & Pazopanib & \\
& Everolimus & \\
\hline
\end{tabular}

Notes: aGrade A recommendation; 'brade B or C recommendation; 'good risk patients only.

Abbreviation: VEGF, vascular endothelial growth factor. remission (PR), and PFS and OS were 4.9 and 14.8 months, respectively. Grounded on these results, everolimus was approved for treatment, after failure of VEGF-targeted agents, in 2009.

Based on the early clinical trials of TKIs in VEGFresistant disease, and some larger retrospective studies, ${ }^{8}$ it remained controversial whether a change of mode of action is mandatory in resistant mRCC. However, the lack of sufficient Phase III data left everolimus the only approved agent in VEGF-resistant disease. Recently, results from the AXIS trial were presented, which showed superior results for axitinib, compared to sorafenib, in pure second line treatment of mRCC. In early 2012, axitinib was approved by the US Food and Drug Administration as a second line option for the treatment of mRCC. This review focuses on axitinib, its clinical development, and its role in the treatment landscape of RCC.

\section{Pharmacology and mode of action}

Axitinib (AG-013736) is a small-molecule indazole derivative, which inhibits the VEGFR, at subnanomolar levels, and its VEGF-mediated endothelial cell proliferation (Table 4). Other targets, such as PDGFR- $\beta$ or c-Kit, require low nanomolar levels of axitinib to achieve receptor inhibition. ${ }^{20,21}$ Blockade of the VEGFR is associated with profound effects on the tumor vasculature in mouse models. A rapid response to axitinib has been observed within 24 hours of treatment, with loss of endothelial sprouts and fenestration in $80 \%$ of tumor vasculature, whereas a different phenotype was induced in remaining vessels. ${ }^{22}$ As a consequence, vascular density was decreased, and VEGFR2 and VEGFR3 expression reduced. However, changes in tumor vasculature remained transient upon cessation of treatment, indicating the reversible nature of target inhibition. ${ }^{23}$ Regrowth of endothelial sprouts was detected within one day after drug withdrawal, and led to complete recovery of tumor vasculature by Day 7 . However, the vessels remained sensitive to another course of axitinib treatment.

Based on compelling evidence, at the molecular level, that inhibition of the VEGFR is associated with regression of tumor vessels, a first-in-human Phase I clinical trial was initiated to evaluate its safety, tolerability, pharmacokinetics (PK), and pharmacodynamics. ${ }^{19,23,24}$ As part of the protocol, the effect of food and antacid co-administration were determined in a sub cohort of patients. A total of 36 patients were treated within the Phase I trial, receiving doses from 5-30 mg twice daily (BID). ${ }^{24}$ Dose-limiting toxicities (DLTs) were reported in eleven patients, consisting of hypertension, hepatic toxicity, seizure, 
Table 2 Clinical outcome of approved first line therapies in metastatic renal cell carcinoma

\begin{tabular}{lllll}
\hline Agent & Poor risk $^{\mathbf{a}}$ & $\begin{array}{l}\text { OS } \\
(\mathbf{m o})\end{array}$ & $\begin{array}{l}\text { PFS first line } \\
(\mathbf{m o})\end{array}$ & $\begin{array}{l}\text { Subsequent } \\
\text { Rx (\%) }\end{array}$ \\
\hline Sunitinib' & No & 26.4 & $\mathrm{II}$ & 56 \\
Bev/IFN $^{2}$ & No & 23.3 & 10.2 & 55 \\
Pazopanib $^{3}$ & No & 22.9 & $11 . \mathrm{I}^{\mathrm{b}}$ & 30 \\
Temsirolimus $^{4}$ & Yes & 10.9 & 5.5 & NA \\
\hline
\end{tabular}

Notes: aAccording to MSKCC risk criteria; bfirst line patients only.

Abbreviations: OS, overall survival; PFS, progression-free survival; Rx, drug prescription; mo, months; NA, not available.

apnea, hemoptysis, stomatitis, pancreatitis, ischemic bowel, thromboembolism, and diarrhea. Because DLT was reached within the first and second cohort of patients, dose de-escalation to $5 \mathrm{mg}$ BID was enforced within the next cohort. The BID dose of $10 \mathrm{mg}$ was considered to be above the maximum tolerated dose (MTD), and was never tested within that study. The MTD and recommended dose were $5 \mathrm{mg}$ BID. Because absorption is best in the fasted state, this was recommended for subsequent Phase II studies.

\section{Pharmacokinetics of axitinib}

Axitinib metabolism is primarily mediated through hepatic elimination, involving cytochrome CYP3A, uridine glucoronosyltransferase, and, to a lesser extent, CYP1A2. Because of potential drug interaction, a subgroup of patients received rabeprazole as a coadministration, but PK was not significantly altered.

A meta-analysis of eleven healthy volunteer studies, with a total of 389 volunteers, explored the role of genetic polymorphism in drug-metabolizing enzymes, but failed to show a significant correlation between polymorphism and PK level. ${ }^{25}$ However, a Phase I study, in patients with mild or moderate hepatic impairment, showed an association between drug exposure and hepatic impairment, indicating the possible need for dose-reductions in these patients. ${ }^{26} \mathrm{The}$ area under the curve (AUC) in patients with normal $(\mathrm{n}=8)$, mild $(n=8)$, and moderate $(n=8)$ liver impairment was 156 , 122 , and $304 \mathrm{ng} \mathrm{h} / \mathrm{mL}$, respectively.
Axitinib is characterized by an oral bioavailability of $58 \%$, and reaches peak concentrations within 2-6 hours after dosing. The terminal plasma half-life is $2-5$ hours, and a steady state is reached within 15 days of treatment. Increasing doses showed a dose-proportional increase of maximum concentration and AUC $(460 \pm 414 \mathrm{ng} \mathrm{h} / \mathrm{mL}) \cdot{ }^{24}$ Axitinib binds strongly to albumin, which corresponds to a plasma protein-binding of more than $99 \%$ (unpublished data). ${ }^{26}$

A partial response was seen in two patients with RCC, and one patient with adenoid cystic carcinoma. Tumor shrinkage or cavitation was seen in patients with mesothelioma, thyroid cancer, RCC, breast cancer, and non-small cell lung-cancer. Based on these promising results, further exploration in subsequent clinical trials was warranted. ${ }^{24}$

\section{Pharmacodynamic marker of axitinib}

Pharmacodynamics are is an important tool to measure biological changes of targeted therapies. With inhibitors of angiogenesis, dynamic contrast-enhanced magnetic resonance imaging (DCE-MRI) has been extensively explored. Morgan et al reported on the use of DCE-MRI as a predictive biomarker for the VEGFR inhibitor PTK/ZK in a Phase I clinical trial. ${ }^{27}$ Later, a pilot study explored DCE-MRI as a putative predictive marker for sorafenib in RCC. ${ }^{28}$ However, a later study tested the area under the contrast concentration versus time curve 90 seconds after contrast injection, and the volume transfer constant of the contrast agent $\left(\mathrm{K}^{\text {trans }}\right)$ in 56 patients, who were prospectively treated with sorafenib. The authors detected biological effects at the start of treatment, but could not validate the predictive nature of the readings, due to high variability within the treatment cohort. ${ }^{29}$

It seems apparent that DCE-MRI is a valuable tool to detect biological alterations based on VEGFR inhibition, but whether these changes predict response in patients remains uncertain. As part of the Phase I trial of axitinib, DCEMRI was explored as a putative pharmacodynamic marker. DCE-MRIs were performed at baseline and at Day 2 of the

Table 3 Subsequent treatment is effective in refractory disease, but studies recruit distinct patient populations, and are not comparable

\begin{tabular}{llllll}
\hline Agent & $\mathbf{n}$ & ORR (\%) & PFS (mo) & OS (mo) & Refractory for \\
\hline Sunitinib $^{7}$ & 61 & 23 & 7.0 & 10.8 & Bevacizumab \\
Axitinib $^{16}$ & 62 & 23 & 7.4 & 13.6 & Sorafenib \\
Sorafenib $^{9}$ & 52 & 10 & NA $^{\mathrm{a}}$ & 7.4 & Sunitinib \\
Temsirolimus $^{17}$ & 29 & $<\mathrm{I}$ & 5.1 & 18.0 & Sunitinib and/or sorafenib \\
Everolimus $^{18, \mathrm{~b}}$ & $4 \mathrm{I}$ & 7 & 11.2 & 22.1 & Cytokines, VEGF targeted agents $^{\mathrm{c}}$ \\
Everolimus $^{5, \mathrm{~b}}$ & 277 & 2 & 4.9 & 14.8 & VEGF-targeted agents \\
\hline
\end{tabular}

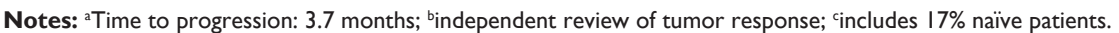

Abbreviations: ORR, overall response rate; PFS, progression-free survival; OS, overall survival; mo, months; NA, not available. 
Table 4 Axitinib inhibitory profile determined by cellular IC50 values $^{20,21}$

\begin{tabular}{ll}
\hline & IC50 (nM) \\
\hline VEGFRI & 1.2 \\
VEGFR2 & $0.25^{\mathrm{a}}$ \\
VEGFR3 & 0.29 \\
PDGF-B & $1.6 / 2.5$ \\
c-KIT & $1.7 / 2.0$ \\
\hline
\end{tabular}

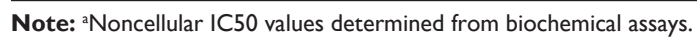

Abbreviations: IC50, half maximum inhibitory concentration; VEGFR, vascular endothelial growth factor receptor; PDGF, platelet-derived growth factor; c-KIT, tyrosine-protein kinase Kit.

first cycle. A linear correlation was found between axitinib exposure and changes in $\mathrm{K}^{\text {trans }}$ and initial AUC. A decrease of $50 \%$ or more in $\mathrm{K}^{\text {trans }}$ indicated vascular response, and corresponded to an AUC $>200 \mathrm{ng} \mathrm{h} / \mathrm{mL} .{ }^{30} \mathrm{DCE}-\mathrm{MRI}$ data suggests that a dose-dependent effect of axitinib on endothelial cells is likely present, but may lack a proportional increase at high exposures.

A class effect of VEGF inhibitors is the development of hypertension, which has been recently proposed as a prognostic and predictive marker for sunitinib treatment in metastatic RCC. ${ }^{31}$ The predictive value of an increase of diastolic blood pressure has been also explored, in a pooled analysis in 230 patients with four different solid tumors, treated with axitinib. ${ }^{32}$ In this mixed-patient population, ORR and median objective response (OR) correlated with a diastolic blood pressure of at least $90 \mathrm{mmHg}$, whereas PFS failed to reach significance. Prospective validation of treatment-induced hypertension is currently being explored, in a prospective, randomized Phase II study, and results are awaited eagerly.

\section{Clinical efficacy of axitinib in $\mathrm{mRCC}$}

The promising preliminary activity of axitinib in $\mathrm{mRCC}$ fostered its development in a pilot Phase II trial, which explored axitinib after failure of cytokine treatment in mRCC. ${ }^{33}$ Patients received $5 \mathrm{mg}$ twice daily axitinib every two days until disease progression or intolerance occurred. Disease response was measured every 8 weeks by investigators. Between October, 2003 and April, 2004, a total of 52 patients received axitinib treatment, of whom 23 (44\%) achieved an OR, including two complete remissions. Early signs of tumor shrinkage were detected in twelve patients, which has been shown to predict time to treatment failure, and OS, in mRCC patients. ${ }^{34}$ Disease stabilization was detected in 22 (42\%) patients, whereas four $(8 \%)$ patients failed to respond to therapy. The time to progression (15.7 months) was extraordinary, and was associated with a promising OS of 29.9 months.
Interestingly, long-term survivorship could be identified in $21 \%$ of patients at 5 years, after extended follow-up. ${ }^{35}$

Based on its clinical efficacy in cytokine-refractory disease, axitinib was explored in RCC patients with failure to sorafenib treatment. The Phase II study explored 62 patients, of whom $18(29 \%)$ failed two or more prior lines of antiangiogenic therapy. All patients failed sorafenib at some point during systemic treatment. ${ }^{16}$ Therapy consisted of axitinib $5 \mathrm{mg}$ BID; dose escalation to $7 \mathrm{mg}$ or $10 \mathrm{mg}$ BID was performed in $33(53 \%)$ patients. PR was achieved in 14 (23\%) patients, and disease stabilization in eleven (18\%) patients, which was associated with a PFS of 7.4 months and an OS of 13.6 months. These results supported the use of axitinib in TKI-refractory RCC, and led to the development of the global AXIS Phase III validation trial in strict second-line therapy.

Between September, 2008 and July, 2010 a total of 723 patients were randomized to receive either axitinib $(\mathrm{n}=361)$ or sorafenib $(\mathrm{n}=362)$ in the Phase III AXIS trial. ${ }^{10}$ Patients were allowed to have received one prior line of therapy only, which may have consisted of cytokines, TKI, bevacizumab, or mTORi. The study was powered to detect an improvement in PFS from 5 to 7 months for axitinib treatment.

Efficacy results showed that second line treatment with axitinib was more effective than sorafenib, as measured by PFS, OR and ORR rates. Through central review, treatment with axitinib was associated with a PFS of 6.7 months, compared to 4.7 months with sorafenib (hazard ratio [HR] $=0.665$ [0.544-0.812]; $P<0.0001)$, and an ORR of 19 and $9 \%$ $(P=0.0001)$, respectively. ${ }^{10}$

Because patients' clinical outcomes vary based on previous therapies, subgroups, by prior therapy with cytokines ( $n=251)$, sunitinib $(n=389)$, bevacizumab $(n=59)$, or temsirolimus $(n=24)$ were explored (Table 5). ${ }^{10,36}$ The best results were gained for both agents in patients with failure of cytokines only. In this subgroup, sorafenib achieved a PFS of 6.5 months, which is superior to historical data from sorafenib's pivotal TARGET trial. However, axitinib improved the PFS, compared to sorafenib, and achieved 12.1 months PFS $(\mathrm{HR}=0.464$ [0.318-0.676]; $P<0.0001)$.

An important aspect of the AXIS trial was the clinical efficacy of axitinib (or sorafenib) after TKI failure. The majority of patients received sunitinib as first-line therapy and, hence, represent the largest subgroup in patients with prior exposure to targeted therapy. For the first time, results from a large Phase III study supported the sequential use of a TKI in second-line therapy. Furthermore, AXIS compared two distinct TKIs head-to-head in a defined scenario. As a consequence 
Table 5 Clinical efficacy by subgroup (AXIS trial)

\begin{tabular}{|c|c|c|c|c|c|c|}
\hline \multirow[t]{2}{*}{ Prior therapy } & \multicolumn{2}{|c|}{ ORR (\%) ${ }^{36}$} & \multirow[t]{2}{*}{$P$-value } & \multicolumn{2}{|l|}{ PFS $(\mathrm{mo})^{10}$} & \multirow[t]{2}{*}{$P$-value } \\
\hline & $\overline{\text { AXI }}$ & $\overline{\text { SOR }}$ & & $\overline{\text { AXI }}$ & SOR & \\
\hline Cytokines & 33 & 14 & 0.0002 & $12.1(10.1-13.9)$ & $6.5(6.3-8.3)$ & $<0.0001$ \\
\hline Sunitinib & 11 & 9 & 0.1085 & $4.8(4.5-6.4)$ & $3.4(2.8-4.7)$ & 0.0107 \\
\hline Bevacizumab & 7 & 3 & 0.2733 & $4.2(2.8-6.5)$ & $4.7(2.8-6.7)$ & 0.6366 \\
\hline Temsirolimus & 42 & 8 & 0.0331 & $10.1(1.5-10.2)$ & $5.3(1.5-10.1)$ & 0.1425 \\
\hline
\end{tabular}

Note: Axitinib improves progression free survival in patients with prior exposure to cytokines or sunitinib.

Abbreviations: ORR, overall survival rate; PFS, progression-free survival; mo, months; AXI, axitinib; SOR, sorafenib.

of prior therapy, prolonged VEGFR inhibition, by either axitinib or sorafenib, achieved a PFS of 4.8 and 3.4 months, respectively $(\mathrm{HR}=0.741[0.573-0.958] ; P=0.0107)$, again underscoring the moderate, but significant, superior efficacy of axitinib in sunitinib-refractory patients. However, results from the bevacizumab and temsirolimus subgroups remain inconclusive, mainly based on the small number of patients treated. A limitation is shared by the RECORD-1 trial, which included only $9 \%$ of patients with failure after bevacizumab treatment. ${ }^{37}$ Currently, the best choice of treatment after either bevacizumab or temsirolimus remains still undefined.

\section{Safety and tolerability in $\mathrm{MRCC}$}

With the introduction to the clinic of specific and potent inhibitors of the VEGFR, such as axitinib or tivozanib, it was generally perceived that specific inhibition may result in a decrease of adverse events and, hence, boost the treatment's tolerability. AXIS is the first trial to report on a direct comparison of two distinct TKIs targeting VEGFR. Discontinuation of treatment due to adverse events remained low in both groups. Treatment with axitinib was associated with a $4 \%$ discontinuation rate, with fatigue and transient ischemic attack being the most common adverse events leading to discontinuation. However, sorafenib was discontinued in $8 \%$ of patients, with hand-foot syndrome, diarrhea, or asthenia as the prevailing adverse events for discontinuation. ${ }^{10}$ One or more dose interruption for any cause was found in $77 \%$ and $80 \%$ of patients, for axitinib and sorafenib treatment, respectively. However, dose reduction was more frequent with sorafenib treatment, and applied to $31 \%$ and $52 \%$ of patients treated with axitinib and sorafenib, respectively. This notion is further supported by the dose escalation of axitinib above $5 \mathrm{mg}$ BID, which was allowed within the trial, and applied to $37 \%$ of patients treated with axitinib.

The spectrum of adverse events has been reported to vary between both compounds. Diarrhea, hypertension, fatigue, anorexia, nausea, and dysphonia remained the prevailing adverse events during axitinib treatment (Table 6). Sorafenib showed a similar range of adverse events, but incidence of certain adverse events varied between compounds. Hypertension, nausea, dysphonia, and hypothyroidism were more frequent with axitinib treatment, whereas hand-foot syndrome, alopecia, and rash were characteristically associated with sorafenib treatment. A similar weight was found among Grade 3 adverse events. In such cases, axitinib treatment expressed hypertension, diarrhea, and fatigue as the most prominent adverse events, whereas sorafenib was associated with hand-foot syndrome, and hypertension.

Hypertension, dysphonia, and hypothyroidism are considered characteristic adverse events of VEGFR inhibitors. ${ }^{38}$ Hypertension is thought to develop through deprivation of endothelial nitric oxide synthesis, upon inhibition of VEGF signalling. ${ }^{39}$ The cause of dysphonia remains unknown, but direct treatment effects at the vocal cords are assumed to be the underlying cause. Development of hypothyroidism has been believed to be a consequence of direct VEGFR inhibition, through induction of thyroiditis, followed by endocrine organ failure. ${ }^{40}$ However, the mechanism to trigger thyroiditis remains elusive.

It seems conceivable that, with the clinical application of pharmacologically more potent VEGFR inhibitors, the incidence of such adverse events increases. Other adverse events, such as hand-foot syndrome and alopecia, are attributable to a distinct tyrosine kinase inhibitor profile, and its incidence may decrease

Table 6 Selected adverse events associated with axitinib treatment in second line ${ }^{10}$

\begin{tabular}{lll}
\hline Adverse events & All grades (\%) & Grade $\geq \mathbf{3}(\%)$ \\
\hline Diarrhea & 55 & $1 \mathrm{I}$ \\
Hypertension & 40 & 16 \\
Fatigue & 39 & $1 \mathrm{I}$ \\
Anorexia & 34 & 5 \\
Nausea & 32 & 3 \\
Dysphonia & 31 & 0 \\
Hand-foot syndrome & 27 & 5 \\
Weight loss & 25 & 2 \\
Hypothyroidism & 19 & 19 \\
Mucosal inflammation & 15 & 1 \\
Rash & 13 & $<1$ \\
\hline
\end{tabular}


with a more selective TKI. These observations certainly need validation, which may be achieved by the pivotal tivozanib trial, comparing this selective VEGFR inhibitor with sorafenib. Overall, the treatment with axitinib was well-tolerated, and no new safety signal was raised in the pivotal Phase III trial.

\section{Conclusion: place of therapy algorithm}

Previous early clinical trials, and the pivotal AXIS trial, establish axitinib as a vital second line option in $\mathrm{mRCC}$, with clinical activity superior to sorafenib. However, how these data compare to everolimus - the approved treatment for refractory $\mathrm{mRCC}$ - remains unknown. Because everolimus was tested in patients resistant to VEGF inhibitors, with multiple lines of prior therapy, patient selection differed substantially from the AXIS trial, where multiple agents were allowed, but prior lines of therapy were restricted to one only. Ongoing trials explore everolimus in strict second-line therapy, which may deliver data more comparable to the AXIS trial. Furthermore, the 404 study compares sorafenib and temsirolimus as second line therapies in $\mathrm{mRCC}$, and may help to define the merits of either of the sequences TKI-TKI or TKI-mTORi in a large randomized trial. However, current retrospective analysis suggests similar outcomes for either sequence. ${ }^{41}$ More importantly, we may have to define subgroups of patients, determined by clinical behavior during the first-line therapy, to define novel treatment algorithms for our patients. Despite the introduction of novel compounds in recent years, patients with intrinsic resistance show a dismal prognosis, ${ }^{42,43}$ and need a distinct approach to treat their disease.

Nevertheless, AXIS has brought the first head-to-head comparison of TKIs and proved that, despite their mutual main target, TKIs may exert distinct clinical activity. For a TKI-based sequential therapy, axitinib is the preferred choice in second line treatment. However, current trials explore axitinib in first line treatment of $\mathrm{mRCC}$, and indicate a putative role for axitinib in the near future.

\section{Disclosure}

The authors report no conflicts of interest in this work.

VG: Honoraria: Pfizer, Novartis, Roche, GSK

Advisory: Pfizer, Novartis, Roche, Bayer

AM: Honoraria: Pfizer, Novartis, Bayer, GSK

Advisory: Bayer, Pfizer

\section{References}

1. Motzer RJ, Hutson TE, Tomczak P, et al. Overall survival and updated results for sunitinib compared with interferon alfa in patients with metastatic renal cell carcinoma. J Clin Oncol. 2009;27(22):3584-3590.
2. Escudier BJ, Pluzanska A, Koralewski P, Ravaud A. Bevacizumab plus interferon alfa-2a for treatment of metastatic renal cell carcinoma: a randomised, double-blind phase III trial. Lancet. 2008;370(9605):2103-2111.

3. Sternberg CN, Davis ID, Mardiak J, et al. Pazopanib in locally advanced or metastatic renal cell carcinoma: results of a randomized Phase III trial. J Clin Oncol. 2010;28(6):1061-1068.

4. Hudes GR, Carducci M, Tomczak P, et al. Temsirolimus, interferon alfa, or both for advanced renal-cell carcinoma. $N$ Engl $J$ Med. 2007;356(22):2271-2281.

5. Motzer RJ, Escudier BJ, Oudard S, et al. Phase 3 trial of everolimus for metastatic renal cell carcinoma: final results and analysis of prognostic factors. Cancer. 2010;116(18):4256-4265.

6. Coppin C, Kollmannsberger C, Le L, Porzsolt F, Wilt TJ. Targeted therapy for advanced renal cell cancer (RCC): a Cochrane systematic review of published randomised trials. BJU Int. 2011;108(10): $1556-1563$.

7. Rini BI, Michaelson MD, Rosenberg JE, et al. Antitumor activity and biomarker analysis of sunitinib in patients with bevacizumab-refractory metastatic renal cell carcinoma. J Clin Oncol. 2008;26(22): 3743-3748.

8. Porta C, Procopio G, Cartenì G, et al. Sequential use of sorafenib and sunitinib in advanced renal-cell carcinoma (RCC): an Italian multicentre retrospective analysis of 189 patient cases. BJU Int. 2011;108(8 Pt 2): E250-E257.

9. Di Lorenzo G, Cartenì G, Autorino R, et al. Phase II study of sorafenib in patients with sunitinib-refractory metastatic renal cell cancer. $J$ Clin Oncol. 2009;27(27):4469-4474.

10. Rini BI, Escudier B, Tomczak P, et al. Comparative effectiveness of axitinib versus sorafenib in advanced renal cell carcinoma (AXIS): a randomised phase 3 trial. Lancet. 2011;378(9807):1931-1939.

11. Linehan WM, Walther MM, Zbar B. The genetic basis of cancer of the kidney. J Urol. 2003;170(6 Pt 1):2163-2172.

12. Zhuang Z, Gnarra JR, Dudley CF, Zbar B, Linehan WM, Lubensky IA. Detection of von Hippel-Lindau disease gene mutations in paraffinembedded sporadic renal cell carcinoma specimens. Mod Pathol. 1996;9(8):838-842.

13. Iliopoulos O, Kibel A, Gray S, Kaelin WG. Tumour suppression by the human von Hippel-Lindau gene product. Nat Med. 1995;1(8):822-826.

14. Patard J-J, Pignot G, Escudier B, et al. ICUD-EAU international consultation on kidney cancer 2010: treatment of metastatic disease. Eur Urol. 2011;60(4):684-690.

15. Ljungberg B, Cowan NC, Hanbury DC, et al. EAU guidelines on renal cell carcinoma: the 2010 update. Eur Urol. 2010;58(3):398-406.

16. Rini BI, Wilding G, Hudes G, et al. Phase II study of axitinib in sorafenib-refractory metastatic renal cell carcinoma. J Clin Oncol. 2009;27(27):4462-4468.

17. Weikert S, Kempkensteffen C, Busch J, et al. Sequential mTOR inhibitor treatment with temsirolimus in metastatic renal cell carcinoma following failure of VEGF receptor tyrosine kinase inhibitors. World J Urol. 2011. [Epub ahead of print.]

18. Amato RJ, Jac J, Giessinger S, Saxena S, Willis JP. A phase 2 study with a daily regimen of the oral mTOR inhibitor RAD001 (everolimus) in patients with metastatic clear cell renal cell cancer. Cancer. 2009;115(11):2438-2446.

19. Mackenzie MJ, Rini BI, Elson P, et al. Temsirolimus in VEGF-refractory metastatic renal cell carcinoma. Ann Oncol. 2011;22(1):145-148.

20. Chow LQM, Eckhardt SG. Sunitinib: from rational design to clinical efficacy. J Clin Oncol. 2007;25(7):884-896.

21. Wickman G, Hallin M, Dillon R, et al. Further characterization of the potent VEGF/PDGF receptor tyrosine kinase inhibitor, AG013736, in preclinical tumor models for its antiangiogenesis and antitumor activity. Proc Am Assoc Cancer Res. 2003;44:A3780.

22. Inai T, Mancuso M, Hashizume H, et al. Inhibition of vascular endothelial growth factor (VEGF) signaling in cancer causes loss of endothelial fenestrations, regression of tumor vessels, and appearance of basement membrane ghosts. Am J Pathol. 2004;165(1):35-52. 
23. Mancuso MR, Davis R, Norberg SM, et al. Rapid vascular regrowth in tumors after reversal of VEGF inhibition. $J$ Clin Invest. 2006;116(10):2610-2621.

24. Rugo HS, Herbst RS, Liu G, et al. Phase I trial of the oral antiangiogenesis agent AG-013736 in patients with advanced solid tumors: pharmacokinetic and clinical results. J Clin Oncol. 2005;23(24): 5474-5483.

25. Brennan M, Williams JA, Chen Y, Tortorici M, Pithavala Y, Liu YC. Meta-analysis of contribution of genetic polymorphisms in drugmetabolizing enzymes or transporters to axitinib pharmacokinetics. Eur J Clin Pharmacol. 2012;68(5):645-655.

26. Tortorici MA, Toh M, Rahavendran SV, et al. Influence of mild and moderate hepatic impairment on axitinib pharmacokinetics. Invest New Drugs. 2011;29(6):1370-1380.

27. Morgan B, Thomas AL, Drevs J, et al. Dynamic contrast-enhanced magnetic resonance imaging as a biomarker for the pharmacological response of PTK787/ZK 222584, an inhibitor of the vascular endothelial growth factor receptor tyrosine kinases, in patients with advanced colorectal cancer and liver metastases: results from two phase I studies. J Clin Oncol. 2003;21(21):3955-3964.

28. Flaherty KT, Rosen MA, Heitjan DF, et al. Pilot study of DCE-MRI to predict progression-free survival with sorafenib therapy in renal cell carcinoma. Cancer Biol Ther. 2008;7(4):496-501.

29. Hahn OM, Yang C, Medved M, et al. Dynamic contrast-enhanced magnetic resonance imaging pharmacodynamic biomarker study of sorafenib in metastatic renal carcinoma. J Clin Oncol. 2008;26(28): 4572-4578.

30. Liu G, Rugo HS, Wilding G, et al. Dynamic contrast-enhanced magnetic resonance imaging as a pharmacodynamic measure of response after acute dosing of AG-013736, an oral angiogenesis inhibitor, in patients with advanced solid tumors: results from a phase I study. J Clin Oncol. 2005;23(24):5464-5473.

31. Rini BI, Cohen DP, Lu DR, et al. Hypertension as a biomarker of efficacy in patients with metastatic renal cell carcinoma treated with sunitinib. J Natl Cancer Inst. 2011;103(9):763-773.

32. Rini BI, Schiller JH, Fruehauf JP, et al. Diastolic blood pressure as a biomarker of axitinib efficacy in solid tumors. Clin Cancer Res. 2011;17(11):3841-3849.

33. Rixe O, Bukowski RM, Michaelson MD, et al. Axitinib treatment in patients with cytokine-refractory metastatic renal-cell cancer: a phase II study. Lancet Oncol. 2007;8(11):975-984.
34. Krajewski KM, Guo M, van den Abbeele AD, et al. Comparison of four early posttherapy imaging changes (EPTIC; RECIST 1.0, tumor shrinkage, computed tomography tumor density, Choi criteria) in assessing outcome to vascular endothelial growth factor-targeted therapy in patients with advanced renal cell carcinoma. Eur Urol. 2011;59(5):856-862.

35. Motzer RJ, de La Motte Rouge T, Harzstark AL, et al. Axitinib second-line therapy for metastatic renal cell carcinoma $(\mathrm{mRCC})$ : Five-year (yr) overall survival (OS) data from a phase II trial. J Clin Oncol. 2011;29(Suppl):Abstr 4547.

36. Escudier B, Loomis AK, Kaprin A, et al. Association of single nucleotide polymorphisms (SNPs) in VEGF pathway genes with progression-free survival (PFS) and blood pressure (BP) in metastatic renal cell carcinoma (mRCC) in the phase 3 trial of axitinib versus sorafenib (AXIS Trial). Eur J Cancer. 2011;47(Suppl 1):Abstr 7103.

37. Motzer RJ, Escudier BJ, Oudard S, et al. Efficacy of everolimus in advanced renal cell carcinoma: a double-blind, randomised, placebocontrolled phase III trial. Lancet. 2008;372(9637):449-456.

38. Grünwald V, Soltau J, Ivanyi P, Rentschler J, Reuter CWM, Drevs J. Molecular targeted therapies for solid tumors: management of side effects. Onkologie. 2009;32(3):129-138.

39. Horowitz JR, Rivard A, van der Zee R, et al. Vascular endothelial growth factor/vascular permeability factor produces nitric oxidedependent hypotension. Evidence for a maintenance role in quiescent adult endothelium. Arterioscler Thromb Vasc Biol. 1997;17(11): 2793-2800.

40. Mukohara T, Nakajima H, Mukai H, et al. Effect of axitinib (AG-013736) on fatigue, thyroid-stimulating hormone, and biomarkers: a phase I study in Japanese patients. Cancer Sci. 2010;101(4):963-968.

41. Busch J, Seidel C, Kempkensteffen C, et al. Sequence therapy in patients with metastatic renal cell carcinoma: comparison of common targeted treatment options following failure of receptor tyrosine kinase inhibitors. Eur Urol. 2011;60(6):1163-1170.

42. Busch J, Seidel C, Weikert S, et al. Intrinsic resistance to tyrosine kinase inhibitors is associated with poor clinical outcome in metastatic renal cell carcinoma. BMC Cancer. 2011;11(1):295.

43. Heng DYC, Xie W, Bjarnason GA, et al. Progression-free survival as a predictor of overall survival in metastatic renal cell carcinoma treated with contemporary targeted therapy. Cancer. 2011;117(12): 2637-2642.
OncoTargets and Therapy

\section{Publish your work in this journal}

OncoTargets and Therapy is an international, peer-reviewed, open access journal focusing on the pathological basis of all cancers, potential targets for therapy and treatment protocols employed to improve the management of cancer patients. The journal also focuses on the impact of management programs and new therapeutic agents and protocols on

\section{Dovepress}

patient perspectives such as quality of life, adherence and satisfaction The manuscript management system is completely online and includes a very quick and fair peer-review system, which is all easy to use. Visit http://www.dovepress.com/testimonials.php to read real quotes from published authors. 